\title{
Prevalence and Clinical Characteristics of Asymptomatic Pyuria in Chronic Kidney Disease
}

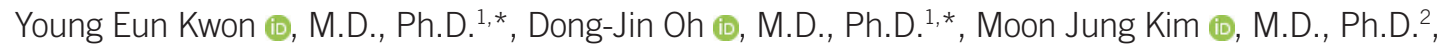 \\ and Hye Min Choi $\mathbb{D}^{0}$, M.D., Ph.D. ${ }^{1}$ \\ ${ }^{1}$ Department of Internal Medicine, Myongji Hospital, Hanyang University College of Medicine, Goyang, Korea; ${ }^{2}$ Department of Laboratory Medicine, Myongji \\ Hospital, Hanyang University College of Medicine, Goyang, Korea
}

Background: Pyuria seems to be common in chronic kidney disease (CKD), irrespective of urinary tract infection (UTI). It has been hypothesized that sterile pyuria occurs in CKD because of chronic renal parenchymal inflammation. However, there are limited data on whether CKD increases the rate of pyuria or how pyuria in CKD should be interpreted. We investigated the prevalence and characteristics of asymptomatic pyuria (ASP) in CKD via urinary white blood cell (WBC) analysis.

Methods: Urine examination was performed for all stable hemodialysis (HD) and non-dialysis CKD patients of the outpatient clinic (total $N=298$ ). Patients with infection symptoms or recent history of antibiotic use were excluded. Urine culture and WBC analysis were performed when urinalysis revealed pyuria.

Results: The prevalence of ASP was 30.5\% (24.1\% in non-dialysis CKD and $51.4 \%$ in HD patients). Over $70 \%$ of the pyuria cases were sterile. The majority of urinary WBCs were neutrophils, even in sterile pyuria. However, the percentage of neutrophils was significantly lower in sterile pyuria. In multivariate logistic regression analysis, the degree of pyuria, percentage of neutrophils, and presence of urinary nitrites remained independently associated with sterile pyuria.

Conclusions: The prevalence of ASP was higher in CKD patients and increased according to CKD stage. Most ASP in CKD was sterile. Ascertaining the number and distribution of urinary WBCs may be helpful for interpreting ASP in CKD.

Key Words: Asymptomatic pyuria, Chronic kidney disease, Cellular analysis, Prevalence, Sterile, Neutrophil
Received: May 15, 2019

Revision received: July 3, 2019

Accepted: November 18, 2019

\section{Corresponding author:}

Hye Min Choi, M.D., Ph.D.

Division of Nephrology, Department of Internal Medicine, Myongji Hospital, Hanyang University College of Medicine, 55 Hwasu-ro 14beon-gil, Deogyang-gu, Goyang 10475, Korea

Tel: +82-31-810-6030

Fax: +82-31-969-0500

E-mail: Itriver@hanmail.net

*These authors equally contributed to this study.

\section{INTRODUCTION}

Pyuria is a useful marker for assessing urinary tract infection (UTI) in the general population. The presence of pyuria is, in general, highly suggestive of UTI, especially in symptomatic patients. Pyuria seems to be common in patients with chronic kidney disease (CKD). Sterile pyuria is considered to occur in CKD owing to chronic renal parenchymal inflammation [1, 2]. However, there are limited data on whether CKD increases the rate of pyuria or how pyuria in CKD should be interpreted [2]. In fact, to our knowledge, the prevalence or characteristics of pyuria has never been investigated, especially in non-dialysis CKD patients.

Studies have addressed the diagnostic value of pyuria in CKD patients, particularly those undergoing dialysis. The prevalence of pyuria in dialysis patients has been reported to range from $31 \%$ to $72 \%$ [1-6], and the rate of documented UTI in these patients ranges from $25 \%$ to $45 \%$. In addition, the presence of 
pyuria may not indicate UTI in dialysis patients $[1,4,5,7]$. Cellular analysis of ascites is useful for early diagnosis of spontaneous bacterial peritonitis in liver cirrhosis patients, and induced sputum analysis provides information on the inflammatory phenotype of airway disease [8, 9]. In the early 1970s, Cabaluna, et al. [1] reported that pyuria is a common finding in hemodialysis (HD) patients that often occurs in the absence of infection. Of interest was the observation that the majority of white blood cells (WBCs) in the urine seemed to be lymphocytes. Unfortunately, no studies have explored urinary cell analysis as a follow-up to these observations of Cabaluna, et al. [1].

We examined whether CKD patients have an increased prevalence of asymptomatic pyuria (ASP) compared with the non-CKD population. In addition, we analyzed urinary WBCs to investigate the characteristics of pyuria and the usefulness of urinary cell analysis in identifying sterile pyuria in the CKD population.

\section{MATERIALS AND METHODS}

\section{Study population and definition}

This single-center, cross-sectional study was performed at the Myongji Hospital, Gyeonggi-do, Korea. The study was approved by the Institutional Review Board of the Myongji Hospital (approval no. MJH15-024), and written informed consent was obtained from all patients.

All HD patients in the dialysis center who voided at least twice every day were included, and urine was obtained before the start of dialysis between September 2016 and March 2017. In addition, stable non-dialysis CKD patients with an estimated glomerular filtration rate (eGFR) $<60 \mathrm{~mL} / \mathrm{min} / 1.73 \mathrm{~m}^{2}$, who visited the outpatient clinic during the study period, were included. eGFR was calculated based on the Chronic Kidney Disease-Epidemiology Collaboration (CKD-EPI) equation [10]. Non-dialysis CKD was divided into stages 3 (eGFR 30-59 mL/min/1.73 m²), 4 (eGFR $15-29 \mathrm{~mL} / \mathrm{min} / 1.73 \mathrm{~m}^{2}$ ), and 5 (eGFR $<15 \mathrm{~mL} / \mathrm{min} / 1.73 \mathrm{~m}^{2}$ ) according to the Kidney Disease Improving Global Outcomes criteria [11].

We excluded patients with evident symptoms of infection, history of antibiotic treatment in the previous month, bladder catheterization, ileal conduit, and combined acute kidney injury. Additionally, patients who did not cooperate were excluded, including those with impaired mobility from whom it was difficult to obtain reliable urine samples.

Overall, the study included 298 patients (179 males, 119 females; median age, 70.5 years [interquartile range (IQR), 57-77 years]). Among them, 70 were HD patients $(51.4 \%$ males, me-

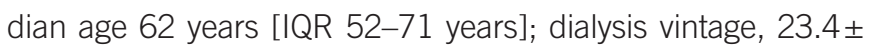
25.9 months), and 228 were non-dialysis CKD patients $(62.7 \%$ males, median age 73 years [IQR 60-78 years]).

For comparison, the prevalence of pyuria in the general population was determined from the data of subjects who received comprehensive health examinations at the same hospital during the study period (total $\mathrm{N}=3,691$; age, $>40$ years; eGFR $\geq 60 \mathrm{~mL}$ $\mathrm{min} / 1.73 \mathrm{~m}^{2}$ ). All CKD patients included in the study, except five, were aged $>40$ years. The median age of the general population (2,489 males, 1,202 females) was 51 years (IQR 45-58 years).

\section{Measurements and definitions}

Clean-catch midstream urine was obtained and processed within one hour of voiding. Urinalysis and urine protein:creatinine ratio (UPCR) were examined for the entire study population. Briefly, fresh urine samples were first examined using an automated analyzer (Cobasu411, Roche Diagnostics, Basel, Switzerland), and parameters such as specific gravity, $\mathrm{pH}, \mathrm{WBC}$, and presence of urinary nitrites were recorded. Next, $10 \mathrm{~mL}$ of urine was centrifuged ( $400 \times g$ for 5 minutes), and the sediment was resuspended in $0.5 \mathrm{~mL}$ of the urine supernatant for microscopic examination. Pyuria was defined as $\geq 5$ WBCs per high-power field (HPF) by urine microscopy of centrifuged urine, which is still considered the standard method [12] despite recent progress in automated urinalysis technology [13]. The number of urinary WBCs were counted as 0-2, 3-5, 6-9, 10-19, 20-29, $30-49,50-99$, and $\geq 100$. Presence of urinary nitrites was determined using urine dipstick analysis.

In this study, urine culture was performed in 86 of the 91 patients with pyuria. Urine culture was performed on MacConkey agar, blood agar, and enrichment media using the platinum loop method. Colony was identified using the Vitek system (bioMerieux, Durham, NC, USA). For urinary WBC analysis, if pyuria was confirmed, $100-200 \mu \mathrm{L}$ of undiluted urine were cytospun onto glass slides using a Cytospin centrifuge (Cellspin, Hanil, Gimpo, Korea) $(400 \times g$ for 5 minutes). Next, differential cell count was analyzed by microscopy ( $\times 400$ fields) following Wright stain.

Pyuria with significant bacteriuria ( $\geq 10^{5}$ colony forming units $[\mathrm{CFU}] / \mathrm{mL}$ ) was defined as UTI. Pyuria without significant bacteriuria was defined as sterile pyuria. Hematuria was defined as the detection of red blood cells at $\geq 5 / \mathrm{HPF}$ in microscopy, and proteinuria was defined as UPCR $\geq 1$.

For serum chemistry, peripheral blood was collected into sterile SST vacuum tubes, centrifuged at 3,000 $\times g$ for 8 minutes, and analyzed using ADVIA Chemistry XPT (Siemens Healthcare Diagnostics, Tokyo, Japan). 


\section{Statistical analysis}

SPSS version 20 (IBM Corp, NY, USA) was mainly used for statistical analyses. Data were presented as means with standard deviation (SD) or as absolute numbers with percentages. Groups were compared using the Student's $t$-test, Mann-Whitney U test, or $\chi^{2}$ test, as appropriate. Logistic regression analyses were conducted to evaluate UTI predictors and determine the role of urinary cell analysis in pyuric patients. Odds ratio (OR) was reported at 95\% confidence interval $(\mathrm{Cl})$. Multivariate logistic regression was performed on variables with $P<0.05$ in univariate analysis (sex, HD, degree of pyuria, percentage of neutrophils, and presence of nitrites) and age.

The utility of the number and distribution of urinary WBCs in UTI detection was assessed by ROC curve analysis using MedCalc Software (version 18.10.2, Ostend, Belgium). Pyuria was further grouped as 6-9, 10-19, 20-49, and $\geq 50$ WBCs/HPF. $P<0.05$ was considered statistically significant.

\section{RESULTS}

\section{ASP prevalence}

The overall ASP prevalence was 30.5\% (18.4\% in males and $48.7 \%$ in females). As shown in Fig. 1, ASP prevalence increased with CKD stage. It was $51.4 \%$ (36.1\% in males and $67.6 \%$ in females) in the $70 \mathrm{HD}$ patients and $24.1 \%(14.0 \%$ in males and

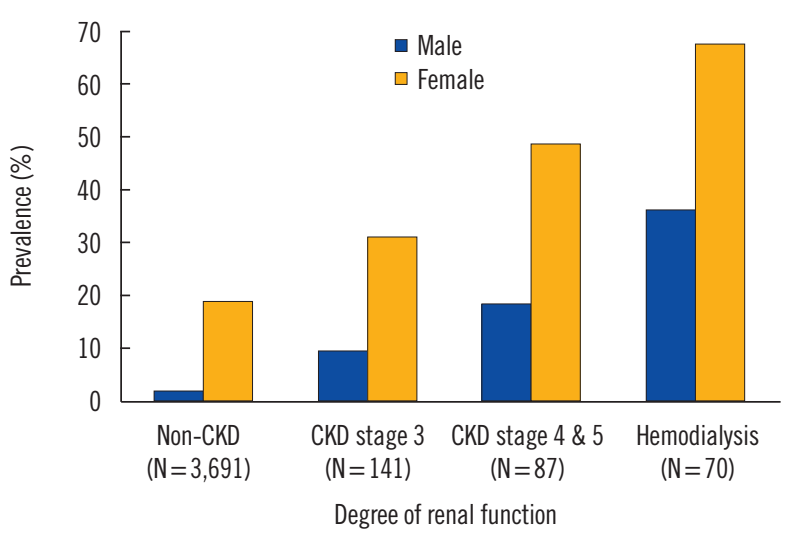

Fig. 1. The prevalence of pyuria according to the level of renal function. The prevalence of pyuria was investigated in asymptomatic CKD population. CKD was divided into HD and non-dialysis CKD; non-dialysis CKD was further classified as stages 3, 4, and 5 according to the KDIGO classification [11]. The prevalence of pyuria in the non-CKD population was estimated from regular health examination data at the study hospital and included control patients aged $>40$ years with eGFR $\geq 60 \mathrm{~mL} / \mathrm{min} / 1.73 \mathrm{~m}^{2}$.

Abbreviations: CKD, chronic kidney disease; eGFR, estimated glomerular filtration rate; HD, hemodialysis; KDIGO, Kidney Disease Improving Global Outcomes.
$41.2 \%$ in females) in the 228 non-dialysis CKD patients.

Pyuria prevalence in the general population was $7.2 \%(1.6 \%$ in males and $18.7 \%$ in females) (Supplemental Data Table S1).

\section{Characteristics of pyuric patients}

Next, we compared the baseline and clinical characteristics of pyuric and non-pyuric CKD patients. As in the general population, pyuria was more common in female than in male patients (Table 1). The pyuric group had more advanced stages of CKD compared with the non-pyuric group. In addition, co-morbid diabetes was more common (64.8\% vs. 48.3\%, $P=0.011$ ), high sensitivity $\mathrm{C}$-reactive protein (hs $\mathrm{CRP}$ ) was higher, $\mathrm{Hb}$ and albumin (Alb) levels were lower, and hematuria and proteinuria were more common in the pyuric group than in the non-pyuric group. Median age did not differ between the pyuric and non-pyuric groups.

Table 1. Baseline and clinical characteristics of the patients with pyuria

\begin{tabular}{lccr}
\hline & $\begin{array}{c}\text { Non-Pyuria } \\
(\mathrm{N}=207)\end{array}$ & \multicolumn{1}{c}{$\begin{array}{c}\text { Pyuria } \\
(\mathrm{N}=91)\end{array}$} & \multicolumn{1}{c}{$P$} \\
\hline Male sex, N (\%) & $146(70.5)$ & $33(36.3)$ & $<0.001$ \\
Age (yr) & $70(57-77)$ & $71(59-77)$ & 0.801 \\
Cause of CKD, N (\%) & & & 0.025 \\
$\quad$ Diabetes & $87(46.8)$ & $54(65.9)$ & \\
Hypertension & $48(25.8)$ & $11(13.4)$ & \\
Glomerulopathy & $23(12.4)$ & $6(7.3)$ & \\
Others & $28(15.1)$ & $11(13.4)$ & \\
Comorbid diabetes, N (\%) & $100(48.3 \%)$ & $59(64.8 \%)$ & 0.011 \\
CKD stage* ${ }^{*}$ (\%) & & & $<0.001$ \\
Stage 3 & $115(55.6)$ & $26(28.6)$ & \\
Stages 4 and 5 & $58(28.0)$ & $29(31.9)$ & \\
HD & $34(16.4)$ & $36(39.6)$ & \\
Presence of hematuria, N (\%) & $32(15.5)$ & $29(31.9)$ & 0.002 \\
Presence of proteinuria, N (\%) & $92(44.7)$ & $59(64.8)$ & 0.002 \\
Laboratory findings (blood) & & & \\
Hb (g/L) & $118.1 \pm 20.2$ & $109.4 \pm 16.2$ & $<0.001$ \\
WBC (×10\%/L) & $6.8 \pm 1.9$ & $6.8 \pm 1.9$ & 0.821 \\
hsCRP (nmol/L) & $18.1 \pm 22.8$ & $33.3 \pm 52.4$ & 0.012 \\
Alb (g/L) & $40.1 \pm 3.2$ & $38.4 \pm 4.3$ & $<0.001$ \\
\hline
\end{tabular}

Values are expressed as mean \pm SD, median (IQR), or number (\%). ${ }^{*} \mathrm{CKD}$ was divided into HD and non-dialysis CKD; non-dialysis CKD was further classified as stage 3, 4, and 5, according to the KDIGO classification [11]. Abbreviations: CKD, chronic kidney disease; WBC, white blood cell; HD, hemodialysis; hsCRP, high sensitivity C-reactive protein; Alb, albumin; IQR, Interquartile range; KDIGO, Kidney Disease Improving Global Outcomes. 


\section{Sterile pyuria and UTI}

Among the 86 ASP patients who were further analyzed by urine culture, 67 (77.9\%) were confirmed as sterile pyuria (88.9\% of HD and $70.0 \%$ of non-dialysis CKD patients). The majority of the bacteria (78.9\%) were Escherichia coli. Next, we compared various parameters between the sterile pyuria and UTI groups. Male patients, higher stages of CKD, fewer urinary WBCs, and absence of urinary nitrites were associated with sterile pyuria (Table 2).

Contrary to our expectations that lymphocytes would be the

Table 2. Baseline and clinical characteristics according to the presence of bacteriuria in the patients with pyuria

\begin{tabular}{|c|c|c|c|}
\hline & $\begin{array}{l}\text { Sterile pyuria } \\
\qquad(\mathrm{N}=67)\end{array}$ & $\begin{array}{c}\text { UTI } \\
(N=19)\end{array}$ & $P$ \\
\hline Male sex, N (\%) & $30(44.8)$ & $2(10.5)$ & 0.007 \\
\hline Age (yr) & $68(57-77)$ & $74(64-78)$ & 0.164 \\
\hline Presence of diabetes, $\mathrm{N}(\%)$ & $44(65.7)$ & $13(68.4)$ & 1.000 \\
\hline Stage of CKD*, N (\%) & & & 0.049 \\
\hline Stage 3 & $18(26.9)$ & $5(26.3)$ & \\
\hline Stages 4 and 5 & $17(25.4)$ & $10(52.6)$ & \\
\hline$H D$ & $32(47.8)$ & $4(21.1)$ & \\
\hline \multicolumn{4}{|l|}{ Blood } \\
\hline $\mathrm{Hb}(\mathrm{g} / \mathrm{L})$ & $108.9 \pm 16.0$ & $107.7 \pm 14.9$ & 0.776 \\
\hline $\mathrm{WBC}\left(\times 10^{9} / \mathrm{L}\right)$ & $6.8 \pm 1.9$ & $7.0 \pm 1.6$ & 0.618 \\
\hline hs CRP (nmol/L) & $34.5 \pm 54.9$ & $35.9 \pm 50.8$ & 0.924 \\
\hline Alb $(g / L)$ & $38.1 \pm 3.5$ & $38.4 \pm 5.0$ & 0.825 \\
\hline Urinary WBCs (/HPF), N (\%) & & & 0.002 \\
\hline $5-9$ & $36(53.7)$ & $2(10.5)$ & \\
\hline $10-19$ & $11(16.4)$ & $2(10.5)$ & \\
\hline $20-49$ & $9(13.4)$ & $7(36.8)$ & \\
\hline$\geq 50$ & $11(16.4)$ & $8(42.1)$ & \\
\hline Presence of urinary nitrites, $\mathrm{N}(\%)$ & $3(4.5)$ & $10(52.6)$ & $<0.001$ \\
\hline Presence of proteinuria, N (\%) & $46(68.7)$ & $12(63.2)$ & 0.782 \\
\hline Presence of hematuria, N (\%) & $25(37.3)$ & $4(21.1)$ & 0.273 \\
\hline \multicolumn{4}{|l|}{ Urinary cell distribution } \\
\hline Neutrophils (\%) & $70.2 \pm 29.2$ & $92.8 \pm 10.1$ & $<0.001$ \\
\hline Lymphocytes (\%) & $18.8 \pm 24.8$ & $4.7 \pm 9.1$ & 0.001 \\
\hline Monocytes (\%) & $9.9 \pm 12.0$ & $2.1 \pm 2.0$ & $<0.001$ \\
\hline
\end{tabular}

Values are expressed as mean $\pm S D$, median (IQR), or number (\%).

${ }^{*}$ CKD was divided into HD and non-dialysis CKD; non-dialysis CKD was further classified as stages 3, 4, and 5, according to the KDIGO classification [11].

Abbreviations: UTI, urinary tract infection; CKD, chronic kidney disease; WBC, white blood cell; hs CRP, high sensitivity C-reactive protein; Alb, albumin; HPF, high-power field; HD, hemodialysis; IQR, interquartile range; KDIGO, Kidney Disease Improving Global Outcomes. predominant WBCs, most urinary WBCs were neutrophils, even in the sterile pyuria group. However, the percentage of neutrophils was significantly lower $(70.2 \%$ vs. $92.8 \%, P<0.001)$ and the percentage of lymphocytes and monocytes was significantly higher ( $18.8 \%$ vs. $4.7 \%, P=0.001$ and $9.9 \%$ vs. $2.1 \%, P<0.001$, respectively) in sterile pyuria group compared with UTI group.

In particular, all pyuria cases with neutrophils accounting for $<60 \%$ of the urinary WBCs were sterile. Furthermore, neutrophils accounted for $\geq 90 \%$ of the urinary WBCs in all bacteriuria cases, except two, which showed $61 \%$ and $72 \%$ neutrophils.

\section{Logistic regression analysis}

In univariate analysis, sex, CKD classification, the number and distribution of urinary WBCs, and presence of urinary nitrites were significant parameters for UTI (Table 3). In multivariate analysis, the degree of pyuria, percentage of neutrophils, and presence of urinary nitrites remained independently associated with higher odds of UTI.

\section{ROC curve}

We evaluated the diagnostic performance of the number of urinary WBCs and the percentage of neutrophils in UTI identification using ROC curves (Fig. 2).

The number of urinary WBCs detected in UTI with an area under the curve (AUC) of 0.778 (95\% $\mathrm{Cl} 0.661-0.870, P<0.001$, and had a sensitivity of $79.0 \%$, and a specificity of $70.2 \%$ at a

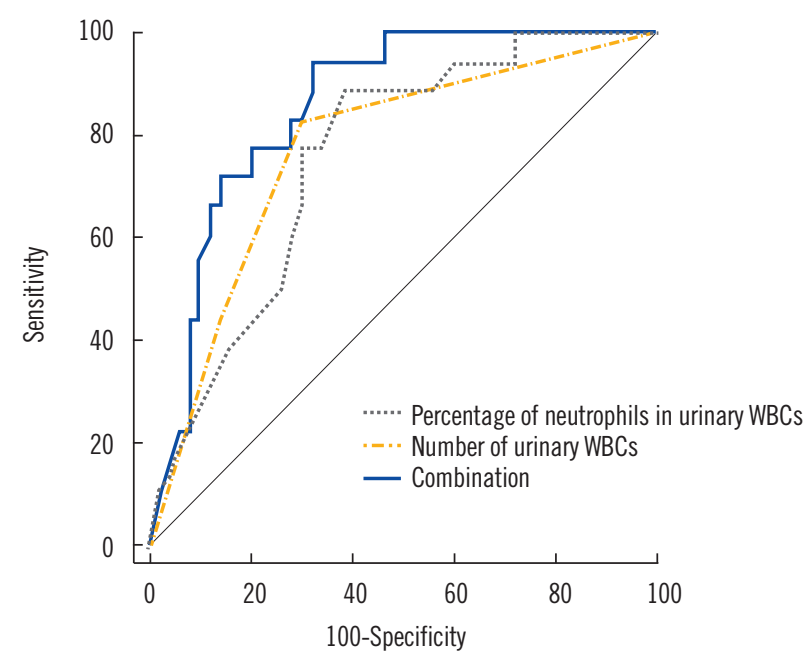

Fig. 2. ROC curves showing the effectiveness of the number and distribution of urinary WBCs in UTI identification. The number of WBCs was analyzed at different cut-off values: $\geq 10, \geq 20$, and $\geq 50$ WBCs/HPF.

Abbreviations: ROC, receiver operating characteristic; UTI, urinary tract infection; WBC, white blood cells; HPF, high-power field. 
Table 3. Univariate and multivariate analyses for predicting UTI in patients with pyuria $(\mathrm{N}=86)$

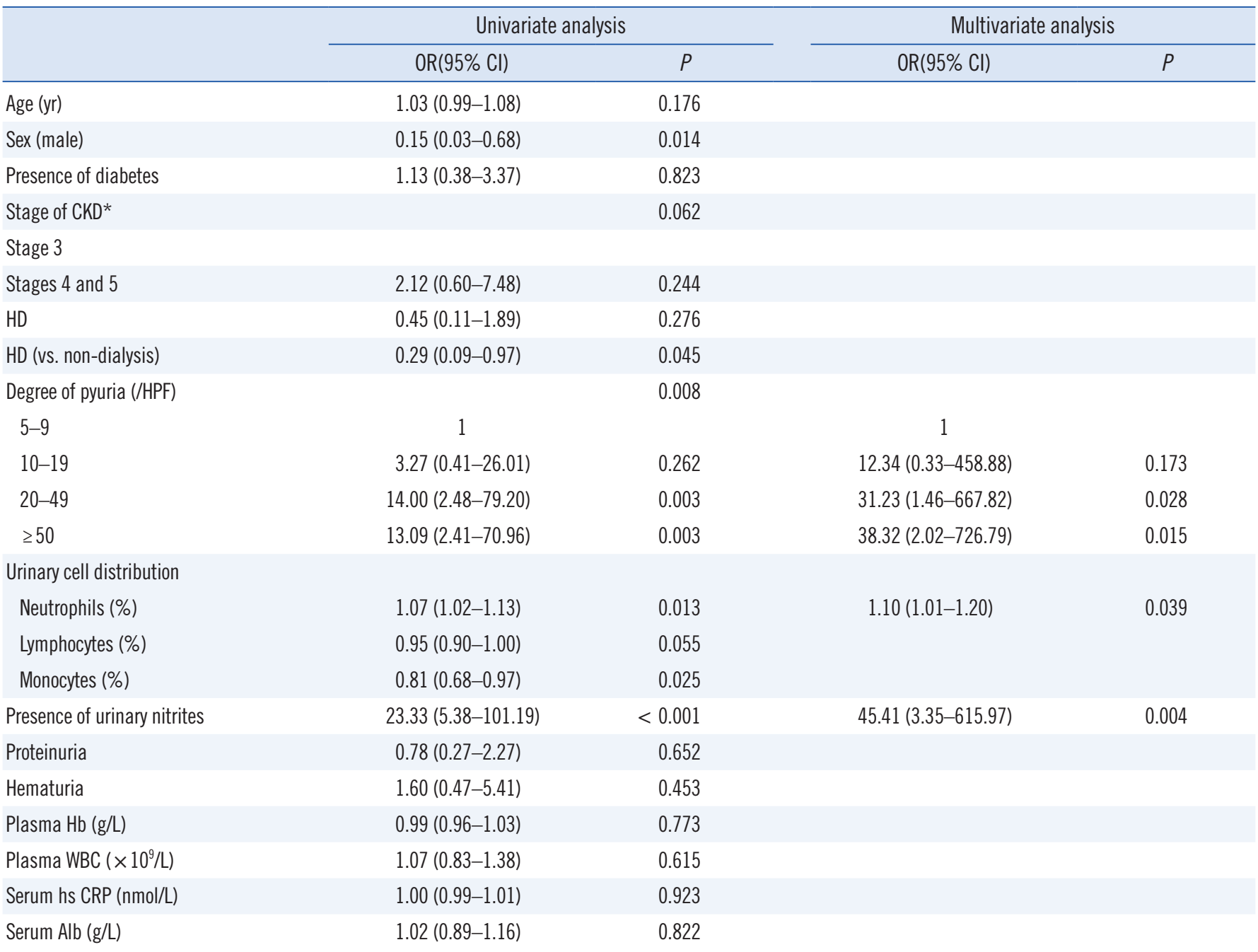

${ }^{*}$ CKD was divided into HD and non-dialysis CKD; non-dialysis CKD was further classified as stage 3, 4, and 5, according to the KDIGO classification [11]. Abbreviations: UTI, urinary tract infection; CKD, chronic kidney disease; HPF, high-power field; WBC, white blood cell; hs CRP, high sensitivity C-reactive protein; Alb, albumin; HD, hemodialysis; OR, odds ratio; $\mathrm{Cl}$, confidence interval; KDIGO, Kidney Disease Improving Global Outcomes.

cutoff value of 20/HPF. The percentage of neutrophils detected UTI with an AUC of $0.759(95 \% \mathrm{Cl} 0.640-0.854, P<0.001)$, with a sensitivity of $88.9 \%$, and a specificity of $62.0 \%$ at a cutoff value of $88 \%$. The combination of both urinary factors showed increased diagnostic performance, with an AUC of 0.857 (95\% Cl 0.751-0.930, $P<0.001)$.

\section{DISCUSSION}

The CKD population had higher ASP prevalence (in both male and female patients) than the non-CKD population, and it increased with CKD stage. Pyuria was more common in female patients and patients with diabetes, higher stages of CKD, proteinuria, hematuria, lower $\mathrm{Hb}$ and $\mathrm{Alb}$, and higher CRP. Over $70 \%$ of the pyuria cases were sterile, and the majority of urinary WBCs were neutrophils, even in sterile pyuria. However, the percentage of neutrophils was significantly lower in sterile pyuria group than in UTI group (70.2\% vs. $92.8 \%$ ). In multivariate logistic regression analysis, the degree of pyuria, percentage of neutrophils, and presence of urinary nitrites were independently associated with sterile pyuria.

In general, CKD patients present with many comorbidities, including diabetes and neurogenic bladder that contribute to bladder stasis, thereby increasing the risk of UTI. Data on the prevalence of UTI among CKD patients is limited, although some authors have suggested that the frequency of UTI might not differ from that in the general population [14]. In the present study, the prevalence of bacteriuria was $6.6 \%(1.1 \%$ in males and 
$14.3 \%$ in females) in the asymptomatic CKD population. We were unable to determine the prevalence of asymptomatic bacteriuria (ASB) in the general population because the regular health examinations did not include a urine culture. A recent meta-analysis showed that diabetic patients have a higher prevalence of ASB than healthy controls (12.2\% vs. 4.5\%) [15].

The diagnosis of UTI in CKD patients can be challenging. UTI symptoms are mostly related to voiding, which is particularly reduced in end-stage renal disease patients. Moreover, it has been suggested that sterile pyuria is common in CKD patients [1, 16]. Our results confirm that ASP is more common in the CKD than in the non-CKD population (24.1\% in non-dialysis CKD and 51.4\% in $\mathrm{HD}$ patients vs. $7.2 \%$ in the non-CKD population). A previous study reported a significantly higher ASP prevalence in both male (12.2\% vs. $3.4 \%$ ) and female (21.4\% vs. $8.7 \%$ ) diabetic patients compared with that in the non-diabetic population [17]. In the present study, ASP was even more common in the CKD population (17.2\% in males and $47.5 \%$ in females) than in the diabetic population. The factors underlying higher ASP prevalence in the CKD population, despite the relatively modest bacteriuria prevalence, are unknown and need to be further examined.

It is noteworthy that $78 \%$ of the pyuria cases were sterile. Overall, $24.2 \%$ of the asymptomatic CKD population (17.3\% males and $34.5 \%$ females) showed sterile pyuria (Supplemental Data Table S2). A previous study reported that $2.6 \%$ males and $13.9 \%$ females have sterile pyuria in the general population; these values were lower than those observed in the CKD patients in this study $[18,19]$. Moreover, the prevalence of sterile pyuria increased with increasing CKD stage (Supplemental Data Table S2). Although we could not fully determine the causes of sterile pyuria in CKD patients, various possibilities exist, including leakage of cells due to tubulointerstital injury.

While it seems that pyuria is common in CKD patients and a low degree of pyuria might be sterile, optimal cut-off values for urinary WBC counts for predicting sterile pyuria in CKD patients have not yet been established. In the present study, pyuria with $\geq 10$ WBCs/HPF was indicative of UTI with high sensitivity (89.5\%) but low specificity (53.7\%), while pyuria with $\geq 20 \mathrm{WBCs} / \mathrm{HPF}$ showed favorable sensitivity (79.0\%) and specificity (70.2\%) for UTI detection. The negative predictive value of urinary WBCs at a cut-off value of 10 WBCs/HPF was $94.7 \%$. In other words, if urinary WBC count was $<10$ WBCs/HPF in asymptomatic CKD patients, the possibility of sterile pyuria is $>90 \%$.

In contrast to our expectation that lymphocytes would account for most of the WBCs, the majority of urinary WBCs were neutrophils, even in sterile pyuria. This finding also contrasted with that of Cabaluna, et al. [1], although they did not report concrete differential cell count results. In this study, a low percentage of urinary neutrophils $(<70 \%)$ was associated with a high probability of sterile pyuria (specificity $94.4 \%$ ). The present findings are valuable as they present an additional tool for predicting sterile pyuria in pyuric CKD patients. Urinary cell analysis may help reduce unnecessary antibiotic administration or suspicion of other factors in the initial stages of asymptomatic infection. Examination of pyuria patterns through urinary cell analysis in more diverse CKD populations might provide valuable information regarding the pathobiological pathways involved in CKD.

This study had several limitations. First, despite the relatively large sample size, this was a single-center study. Second, the criteria for diagnosing UTI vary from study to study. Given that this study included an asymptomatic CKD population, UTI may actually mean ASB. However, originally, the term urinary tract infection encompassed various clinical entities including ASB, cystitis, and pyelonephritis [20]. Indeed, much of the literature concerning UTI does not differentiate between UTI and ASB [20]. Considering that urinary symptoms might be vague in CKD patients, some of the significant bacteriuria accompanying pyuria in this study might have approached clinical infection. Third, the analyses were conducted using a single urine sample from each patient, rather than using a longitudinal follow-up with multiple samples [21]. In addition, we did not examine the presence of urinary tuberculosis (TB), although it is one of the known causes of sterile pyuria. However, we considered the incidence of asymptomatic urinary TB to be low enough to dismiss the possibility.

Despite these limitations, we believe our results are important as we addressed the prevalence of sterile pyuria in an asymptomatic CKD population. To our knowledge, the prevalence of ASP or sterile pyuria has never been investigated according to CKD stage, and this is the first study to perform urinary cell analysis in pyuric CKD patients.

In conclusion, sterile pyuria is common in CKD patients. The number and distribution of urinary WBCs may be helpful for predicting sterile pyuria in CKD patients with pyuria. A large, prospective study with longitudinal follow-up may further clarify the interpretation of urinalysis in the CKD population.

\section{AUTHOR CONTRIBUTIONS}

Young-Eun Kwon: Analysis and interpretation of data, drafting the article, final approval of version to be published. Dong-Jin 
Oh: Analysis and interpretation of data, providing intellectual content of critical importance to the work, revising the article, final approval of version to be published. Moon Jung Kim: Acquisition and analysis of data, providing intellectual content of critical importance to the work, final approval of version to be published. Hye Min Choi: Conception, acquisition, analysis and interpretation of data, revising the article, providing intellectual content of critical importance to the work, final approval of version to be published.

\section{CONFLICTS OF INTEREST}

No potential conflicts of interest relevant to this article were reported.

\section{RESEARCH FUNDING}

Dr. Choi has received funding from Myongji Hospital (faculty grant of Myongji Hospital, 150108).

\section{ORCID}

$\begin{array}{ll}\text { Young-Eun Kwon } & \text { https://orcid.org/0000-0002-0843-9857 } \\ \text { Dong-Jin Oh } & \text { https://orcid.org/0000-0003-1810-7167 } \\ \text { Moon Jung Kim } & \text { https://orcid.org/0000-0003-4148-9116 } \\ \text { Hye Min Choi } & \text { https://orcid.org/0000-0001-8742-4751 }\end{array}$

\section{REFERENCES}

1. Cabaluna CC, Gary NE, Eisinger RP. Urinalysis in patients on chronic hemodialysis. Urology 1977;10:103-4.

2. FasoloLR, Rocha LM, Campbell S, Peixoto AJ. Diagnostic relevance of pyuria in dialysis patients. Kidney Int 2006;70:2035-8.

3. Chaudhry A, Stone WJ, Breyer JA. Occurrence of pyuria and bacteriuria in asymptomatic hemodialysis patients. Am J Kidney Dis 1993;21:180-3.

4. Hyodo T, Yoshida K, Sakai T, Baba S. Asymptomatic hyperleukocyturia in hemodialysis patients analyzed by the automated urinary flow cytometer. Ther Apher Dial 2005;9:402-6.
5. Vij R, Nataraj S, Peixoto AJ. Diagnostic utility of urinalysis in detecting urinary tract infection in hemodialysis patients. Nephron Clin Pract 2009, 113:c281-5.

6. Orłowska A, Majdan M, Kozioł-Montewka M, Grzebalska A, Swatowski A. Asymptomatic bacteriuria in patients on continuous ambulatory peritoneal dialysis. Ann Univ Mariae Curie Sklodowska Med 2002;57:285-9.

7. Oikonomou KG and Alhaddad A. The diagnostic value of urinalysis in hemodialysis patients with fever, sepsis or suspected urinary tract infection. J Clin Diagn Res 2016;10:Oc11-3.

8. Davidson WJ, The S, Leigh R. Establishing a normal range for induced sputum cell counts in Western Canada. Can Respir J 2013;20:424-5.

9. Singh D, Edwards L, Tal-Singer R, Rennard S. Sputum neutrophils as a biomarker in COPD: findings from the ECLIPSE study. Respir Res 2010; 11:77.

10. Levey AS, Stevens LA, Schmid CH, Zhang YL, Castro AF, 3rd, Feldman $\mathrm{HI}$, et al. A new equation to estimate glomerular filtration rate. Ann Intern Med 2009;150:604-12.

11. KDIGO. Chapter 1: Definition and classification of CKD. Kidney Int Suppl 2013;3:19-62.

12. Kim SM, Corwin HL. Urinalysis. In:Schrier RW. ed. Diseases of the kidney. 7th ed. Philadelphia: Lippincott Williams \& Wilkins, 2001:317-31.

13. Oyaert $\mathrm{M}$ and Delanghe J. Progress in automated urinalysis. Ann Lab Med 2019;39:15-22.

14. Gilbert DN. Urinary tract infections in patients with chronic renal insufficiency. Clin J Am Soc Nephrol 2006;1:327-31.

15. Renko M, Tapanainen P, Tossavainen P, Pokka T, Uhari M. Meta-analysis of the significance of asymptomatic bacteriuria in diabetes. Diabetes Care 2011;34:230-5.

16. Eisinger RP, Asghar F, Kolasa C, Weinstein MP. Does pyuria indicate infection in asymptomatic dialysis patients? Clin Nephrol 1997;47:50-1.

17. Ozdem S, Bayraktar T, Oktay C, Sari R, Gültekin M. The prevalence of asymptomatic pyuria in diabetic patients: comparison of the Sysmex UF-100 automated urinalysis analyzer with Fuchs-Rosenthal hemacytometer. Clin Biochem 2006;39:873-8.

18. Alwall $\mathrm{N}$ and Lohi A. A population study on renal and urinary tract diseases. II. Urinary deposits, bacteriuria and ESR on screening and medical examination of selected cases. Acta Med Scand 1973;194:529-35.

19. Wise GJ and Schlegel PN. Sterile pyuria. N Engl J Med 2015;372:104854.

20. Gupta $\mathrm{K}$ and Trautner B. Urinary tract infections, pyelonephritis, and prostatitis. In: Kasper D, Fauci A, et al. eds. Harrison's principles of internal medicine. 19th ed. New York: McGraw-Hill Education, 2015:861-8.

21. Hwang JH, Park HC, Jeong JC, Ha Baek S, Han MY, Bang K, et al. Chronic asymptomatic pyuria precedes overt urinary tract infection and deterioration of renal function in autosomal dominant polycystic kidney disease. BMC Nephrol 2013;14:1. 
Kwon YE, et al.

Asymptomatic pyuria and chronic kidney disease

Supplemental Data Table S1. The prevalence of pyuria according to age

(A) CKD patients

\begin{tabular}{|c|c|c|c|}
\hline Age (yr) & Overall, N (\%) & Male, N (\%) & Female, N (\%) \\
\hline \multicolumn{4}{|l|}{ All CKD } \\
\hline$<60(\mathrm{~N}=86)$ & $26(30.2)$ & $8(14.5)$ & $18(58.1)$ \\
\hline $60-75(N=125)$ & 39 (31.2) & $17(21.5)$ & $22(47.8)$ \\
\hline$\geq 76(N=87)$ & $26(29.9)$ & $8(17.8)$ & 18 (42.9) \\
\hline \multicolumn{4}{|l|}{ Non-dialysis CKD } \\
\hline$<60(\mathrm{~N}=54)$ & 12 (22.2) & $3(7.7)$ & $9(60.0)$ \\
\hline $60-75(\mathrm{~N}=95)$ & 22 (23.2) & $10(15.9)$ & $12(37.5)$ \\
\hline$\geq 76(N=79)$ & $21(26.6)$ & $7(17.1)$ & $14(36.8)$ \\
\hline \multicolumn{4}{|l|}{$H D$} \\
\hline$<60(\mathrm{~N}=32)$ & $14(43.8)$ & $5(31.3)$ & $9(56.3)$ \\
\hline $60-75(\mathrm{~N}=30)$ & $17(56.7)$ & $7(43.8)$ & $10(71.4)$ \\
\hline$\geq 76(\mathrm{~N}=8)$ & $5(62.5)$ & $1(25.0)$ & $4(100.0)$ \\
\hline
\end{tabular}

(B) General population with eGFR $>60 \mathrm{~mL} / \mathrm{min} / 1.73 \mathrm{~m}^{2 *}$

\begin{tabular}{lccr}
\hline Age $(\mathrm{yr})$ & Overall, N (\%) & Male, N (\%) & Female, N (\%) \\
\hline $40-49(\mathrm{~N}=1,655)$ & $119(7.2)$ & $15(1.3)$ & $104(20.6)$ \\
$50-59(\mathrm{~N}=1,221)$ & $80(6.6)$ & $13(1.5)$ & $67(18.2)$ \\
$60-69(\mathrm{~N}=524)$ & $42(8.0)$ & $6(1.9)$ & $36(16.9)$ \\
$\geq 70(\mathrm{~N}=291)$ & $23(7.9)$ & $5(2.8)$ & $18(15.7)$ \\
Total $(\mathrm{N}=3,691)$ & $264(7.2)$ & $39(1.6)$ & $225(18.7)$ \\
\hline
\end{tabular}

The prevalence of pyuria in the general population was obtained from regular health examination data at the study hospital.

*Total $\mathrm{N}=3,691$ (2,489 males, 1,202 females).

Abbreviations: CKD, chronic kidney disease; eGFR, estimated glomerular filtration rate; HD, hemodialysis. 
Supplemental Data Table S2. Prevalence of sterile pyuria in the CKD population

\begin{tabular}{lccc}
\hline & Overall, $\mathbf{N}(\%)$ & Male, $\mathbf{N}(\%)$ & Female, $\mathbf{N}(\%)$ \\
\hline Non-dialysis CKD & & & \\
CKD stage $3(\mathrm{~N}=141)$ & $21(14.9)$ & $9(9.5)$ & $12(26.1)$ \\
CKD stages 4 and $5(\mathrm{~N}=87)$ & $19(21.8)$ & $10(20.8)$ & $9(23.1)$ \\
CKD stages 3,4 , and $5(\mathrm{~N}=228)$ & $40(17.5)$ & $19(13.3)$ & $21(24.7)$ \\
HD ( $\mathrm{N}=70)$ & $32(45.7)$ & $12(33.3)$ & $20(58.8)$ \\
Overall ( $\mathrm{N}=298)$ & $72(24.2)$ & $31(17.3)$ & $41(34.5)$ \\
\hline
\end{tabular}

Abbreviation: CKD, chronic kidney disease; HD, hemodialysis. 